Arts

et Savoirs

\section{Arts et Savoirs}

12 | 2019

Révolution et évolution

\title{
Antediluviana, poème géologique, Ernest Cotty, 1875
}

Antediluviana, geologic poem by Ernest Cotty 1875

\section{Yohann Ringuedé}

\section{(2) OpenEdition}

\section{Journals}

Édition électronique

URL : http://journals.openedition.org/aes/2262

DOI : 10.4000/aes.2262

ISSN : 2258-093X

Éditeur

Laboratoire LISAA

Référence électronique

Yohann Ringuedé, «Antediluviana, poème géologique, Ernest Cotty, 1875 », Arts et Savoirs [En ligne],

12 | 2019, mis en ligne le 25 février 2020, consulté le 24 septembre 2020. URL : http://

journals.openedition.org/aes/2262 ; DOI : https://doi.org/10.4000/aes.2262

Ce document a été généré automatiquement le 24 septembre 2020.

Centre de recherche LISAA (Littératures SAvoirs et Arts) 


\section{Antediluviana, poème géologique, Ernest Cotty, 1875}

Antediluviana, geologic poem by Ernest Cotty 1875

Yohann Ringuedé

\section{Introduction}

1 On sait peu de chose de la vie d'Ernest Paul Cotty. Il naît en 1818 en Essonne (à Montgeron), et meurt en 1877 à Tours. Il mena une carrière militaire à l'étranger qui le conduisit surtout, dans les années 1850, en Afrique du nord. On apprend ainsi, en lisant ses opuscules scientifiques, qu'il séjourna longtemps en Algérie, qu'il participa à la guerre de Crimée, passa à Constantinople, retourna en Algérie (à Maghrnia, proche de la frontière marocaine), participa à une expédition au Maroc et quitta l'Algérie par Oran pour rentrer en France. À son retour, à partir de 1860, il consigna un nombre important de ses observations sur les insectes en tant que membre de la Société entomologique de France et de la Société linnéenne du nord de la France.

2 Il nous a laissé deux textes d'observations entomologiques et biologiques ${ }^{1}$, des notes sur une visite faite au musée d'histoire naturelle de Tours's ${ }^{2}$ deux poèmes d'inspiration scientifique $^{3}$ et quelques poèmes, tantôt légers, tantôt lyriques, parus dans la Revue de la société littéraire, historique et archéologique de l'Ain dont il était correspondant ${ }^{4}$. Ses notes d'entomologie s'appuient à la fois sur des souvenirs d'enfance (notamment liés à des séjours sur l'île d'oléron, en 1830), sur des observations faites durant ses années de service, au Maghreb, mais aussi sur des expéditions effectuées en France après son retour. Son premier poème scientifique, L'Entomologie. Odes sur les coléoptères, paru en 1874, s'inspire directement de ses observations personnelles et loue les beautés anatomiques de cet ordre d'insectes qui comporte le plus grand nombre d'espèces décrites. Dieu est loué à la fin de cette ode dithyrambique de quatre pages comme le «divin lapidaire" responsable de ces joyaux vivants. En 1875, Cotty rédige le long poème Antediluviana, poème géologique, directement inspiré de l'ouvrage à succès du 
vulgarisateur Louis Figuier, La Terre avant le déluge ${ }^{5}$. Comme dans le poème précédent, il mêle science, lyrisme et louange chrétienne.

La figure d'Ernest Cotty est susceptible d'intéresser la recherche (et en particulier, dans le sillage ouvert d'abord par Michel Pierssens, celle qui adopte la méthode épistémocritique) parce qu'elle peut apparaître comme symptomatique des paradoxes du second dix-neuvième siècle vis-à-vis du savoir scientifique, de sa diffusion et de ses rapports avec les lettres. Cotty, militaire de métier, est un autodidacte à la fois en biologie mais aussi en poésie. Ce faisant, il n'est pas sans rappeler des personnalités comme Jean-Henri Fabre ou Élisée Reclus, toutes deux passées à la postérité à la fois pour la teneur scientifique de leurs œuvres en même temps que pour leur valeur poétique. Si Cotty n'a pas été retenu par l'histoire, c'est parce que sa production est bien plus humble, dans sa conception comme dans sa diffusion. Toutefois, il convient de le classer parmi ces figures d'hommes de savoirs qui se sont faits tout seuls. Ils sont les fruits d'une époque qui a rendu les connaissances scientifiques accessibles au populaire, via la vulgarisation, mais a également décomplexé tout un chacun en lui permettant de prendre part, sous la bannière positiviste de l'observation et de l'empirisme, à la construction d'un savoir collectif et à sa diffusion sous des formes revendiquées comme artistiques.

\section{Un « poème géologique » : structure et tonalité}

4 Antediluviana, poème de 588 alexandrins de facture relativement classique, en rimes suivies, est composé en 1875 , à Tours, et paraît en 1876 en fascicule après avoir été édité en deux livraisons dans la Revue de la Société littéraire, artistique et archéologique de l'Ain. Il est sous-titré poème géologique. Le genre n'est pas neuf, puisqu'en 1860, Edmond Emerich fait paraitre un fascicule poétique de quinze pages, en alexandrins de rimes suivies, intitulé $L a$ Création du globe terrestre, sous-titré poème géologique ${ }^{6}$. Le parallèle ne s'arrête pas là, puisque les deux poèmes ont en commun de croiser les connaissances scientifiques en histoire de la terre et de l'évolution biologique à des explications bibliques et religieuses. Dans les deux textes, la genèse et l'évolution sont orchestrées par Dieu.

5 Le titre choisi par Cotty pour orner son poème est un écho à l'œuvre de Figuier. [A]vant le déluge est ainsi traduit littéralement par Antediluviana. Terme très rare en latin ${ }^{7}$, il s'agit très probablement ici d'un adjectif substantivé au neutre pluriel, signifiant littéralement les choses antédiluviennes, ce qui permet à Cotty de suggérer qu'il ne s'occupera pas uniquement de la succession des couches terrestres. La Terre du titre de Figuier est ainsi mise de côté, comme pour suggérer que la géologie n'est peut-être pas le sujet central du poème, malgré son sous-titre. Le choix du latin situe le texte dans une filiation de poèmes cosmologiques évoquant les choses de la nature; cette filiation remonte jusqu'à la tradition antique et, pour le moins, au De rerum natura de Lucrèce.

6 Pour structurer son poème, Cotty suit scrupuleusement les âges géologiques tels que Figuier les expose dans son ouvrage. Le "chant premier" s'ouvre sur un aveu d'impuissance face au projet: la science moderne «[s]'exprime en trop grands mots pour des alexandrins» (v.15). L'humilité de l'autodidacte en poésie se double malicieusement d'une réflexion sur le projet en lui-même, puisque dans le cadre serré de l'alexandrin classique, le poète biologiste va s'efforcer de faire entrer de très 
nombreux noms d'espèces fossiles. Il amoncelle même parfois le nom vernaculaire et la dénomination complète dans la taxonomie de Linné ${ }^{8}$ :

Le Mammouth (Elephas primigenius) ; son

Énorme et blanc squelette au cœur donne un frisson !9

Ce « frisson » face à l'énormité de la bête est renforcé par celui que ressent le poète à faire entrer un tel monstre lexical dans le cadre serré de l'alexandrin classique. Ce nom énorme et redoublé déstructure le vers, affaiblit la césure et repousse à la rime un proclitique inapte, dans la métrique classique, à porter l'accent. On le voit, Cotty n'hésite pas à se montrer espiègle avec la forme de son poème.

Cette remarque vaut également pour la tonalité du texte : l'auteur suggère que les sciences géologique et paléontologique sont trop sérieuses, trop solennelles, pour qu'on prenne la liberté de jouer à les mettre en vers. C'est donc à toute une malicieuse poétique de la prétérition qu'il nous convie. Il confesse d'ailleurs qu'« [i]l convient, même en vers, pour le sujet qu'on traite, / D'oser être technique,... ou de faire retraite.» (v.17-18) Après cet avertissement plaisant, le chant premier aborde rapidement l'époque primitive, qu'en lecteur attentif d'Auguste Comte, le poète confesse ne pouvoir qu'entrevoir. Suivent l'époque de transition, l'époque secondaire et l'époque tertiaire, divisées toutes trois en trois sous-parties. Le « chant second » s'attèle à la description de l'époque quaternaire en sept sections. Cette époque est surtout l'occasion de poser la question du Déluge et de rendre compte des débats autour de la véracité du texte biblique. Cotty cherche à démontrer que le texte divin n'est pas contredit par les sciences géologiques modernes. Enfin, tout comme Figuier qu'il traduit parfois en vers de manière très transparente ${ }^{10}$, Cotty rend les derniers honneurs à Dieu, qui a préparé, peut-être, par le recours au Déluge, la venue de l'homme dans un monde apaisé et débarrassé de ses monstres antédiluviens.

$9 \mathrm{Au}$ nombre des influences qui se lisent en filigrane dans le poème de Cotty et qui viennent infléchir le registre du poème, on peut également compter La Fontaine (dont Cotty imite une fable en $1873^{11}$ ). À travers des reformulations faisant référence à ses apologues, la référence introduit par moments une légèreté de ton.

\section{Génétique et poétique : un poème muséographique de forme romantique}

10 La genèse du poème est complexe. En 1871, Cotty entreprend une visite au Musée d'histoire naturelle de Tours. Le but de cette visite est de rendre compte de la collection et de sa disposition muséographique auprès de la Société linnéenne du nord de la France, qui envisageait d'établir un établissement similaire à Amiens. Dans les lignes de ce compte-rendu, Cotty confesse que dans sa jeunesse il a accompagné des géologues dans leurs expéditions. Enthousiaste face aux découvertes faites, il aurait en sa "juvénile audace qui ne manquait pas cependant d'une certaine grandeur originale " projeté un poème intitulé Antédiluviana, poème géologique, "projet passionnel et chimérique qui, heureusement sans doute, n'a jamais été mis à exécution. ${ }^{12} \mathrm{Si}$ le projet est jugé "chimérique», c'est que Cotty regarde science et poésie comme des corps organiques d'origine résolument différentes: un poème géologique serait tératologique. Partant, «chimérique » est également pris au sens commun d'impossible. Pourtant, contrairement à ce que suggère l'adverbe, Antediluviana, de sept ans postérieur à cette visite, fut à nouveau envisagé au moment où Cotty reprend 
conscience du fait que « cette attrayante et merveilleuse science de la Géologie agrandit les pensées non-seulement du naturaliste qui la cultive avec amour, mais encore du philosophe et même du poëte. $»^{13} C^{\prime}$ 'est donc un projet de jeunesse, un temps écarté, qui est remis sur le métier poétique grâce à l'impulsion de cette visite. En passant, cette note nous apprend que la poésie ne fut pas pour Cotty qu'un passe-temps d'homme vieillissant, mais bien une activité à laquelle il souhaitait déjà s'adonner jeune.

11 L'importance de cette visite pour la création poétique de Cotty est telle que l'on retrouve dans L'Entomologie et dans Antediluviana des expressions, des dispositions d'objets exposés et des images reprises telles quelles de la Description de $1871^{14}$. Les prémices des conceptions poétologiques de Cotty y sont également perceptibles. En effet, au sein de sa description, il prend notamment part au débat sur la dureté phonétique des technolectes: "On voit miroiter au Musée [...] une cinquantaine d' Oiseaux-mouches et de Colibris, ces fleurs célestes et animées qui ont des ailes, que l'on appelle Ténuirostre dans la langue ornithologique, parfois un peu dure, comme toutes les technologies de la science ${ }^{15} »$.

12 En ce qui concerne la versification, force est de constater que, ces inclusions de technolectes mises à part, le vers de Cotty adopte une souplesse relative héritée du Romantisme ${ }^{16}$. Deux analyses permettront de le prouver. Il est question du Rhamphorhynque à propos de l'époque secondaire :

- Quel est cet hiéroglyphe, accusé sur le sable?

Du Ramphorynque c'est la trace irrécusable ;

Aîlé, comme l'est son précédent compagnon,

Cette calligraphie est due à son moignon

D'aîle, ainsi qu'à sa patte et sa traînante queue. ${ }^{17}$

13 L'effet de discordance qui rejette le complément du nom « D'aîle » dans le vers suivant est significatif. Comme dans les poétiques romantiques, les effets de discordance, utilisés avec mesure (ils sont assez rares dans les poèmes de Cotty), sont à interpréter. Ici, le rejet reconduit stylistiquement l'idée de moignon puisque l'â̂le, séparée de son groupe syntaxique, est comme amputée par la versification même. De même, Cotty a recours de temps à autre à des trimètres romantiques ${ }^{18}$. Ainsi, le vers 371, " $\mathrm{Ce}$ phénomène aussi terrible que soudain, » peut être considéré comme tel, puisqu'outre des accents internes sur les syllabes quatre, huit et douze, la césure est tout à fait respectée. Ici aussi, ce choix métrique est sémantiquement fertile. Le " phénomène » en question n'est autre que l'époque glaciaire. La structure accentuelle met significativement l'accent sur les deux adjectifs, «terrible » et "soudain", ce qui renforce la violence de cette glaciation. Par ailleurs, le trimètre ralentit le rythme par multiplication des accents et crée ainsi un fort effet de suspens, puisque le vers suivant annonce que cette longue période doit d'abord se dérouler avant qu'advienne enfin le genre humain.

14 Si l'on met de côté quelques rimes faciles («Géologie » et «Paléonthologie » [v. 13-14], «zoologie » et « ornithologie» [v. 305-306]), des inversions un peu maladroites (« Alors ont disparu des botaniques scènes" [v. 214], "Les savants, du vrai beau justes apologistes » [v. 414]) et un vers faux (v.314), la prosodie de Cotty repose sur une évolution de la métrique classique, loin des révolutions poétologiques qui sont en train de voir le jour par ailleurs dans la production contemporaine ${ }^{19}$. Le poème est d'ailleurs émaillé de références intertextuelles poétiques plus ou moins discrètes, des classiques à Vigny. Il se clôt sur de nombreuses références au texte bilique dont le poète propose 
des versets entiers mis en vers. Par ailleurs, Cotty a lu le poète Jacques Delille, gloire déchue de la poésie scientifique qui fit paraître en 1808 un poème au succès phénoménal, Les Trois règnes de la nature, annoté par Cuvier lui-même. Cotty lui emprunte la description du monde naturel en tableaux (selon une episteme, en termes foucaldiens, plutôt classique et prérévolutionnaire). Des vers delillien comme «Ensemble remontons aux lieux de leurs berceaux [...] Quels sublimes aspects! quels tableaux romantiques $!^{20} »$ sont des sources plus que probables aux formulations suivantes: "Remontons maintenant aux couches éocènes» (v.137), «[...] adorables tableaux » (v. 138), « les émouvants tableaux » (v. 419). Le rapprochement est d'autant plus légitime que d'autres intertextes delilliens sont présents dans Antediluviana ${ }^{21}$.

\section{Un poème évolutionniste ? Du catastrophisme cuviériste à la création continuée}

Influencé par le nationalisme fort de l'armée, un militaire tel que Cotty se plaît à mettre en avant des savants français. Dans la hiérarchie qu'il fait des gloires scientifiques, le premier rang est dédié à ses compatriotes (v. 539-540). Les autres savants loués (v. 544) sont des Britanniques qui sont venus en France consacrer la gloire de Boucher de Perthes en authentifiant ses hypothèses. Quant aux savants allemands, ils sont presque absents $^{22}$ (la blessure de la guerre franco-prussienne l'explique probablement ${ }^{23}$ ). Ce parti-pris, ainsi que celui qui a conduit Cotty à adopter comme source scientifique un ouvrage de vulgarisation français et déjà ancien, explique les particularités de l'histoire de la biologie telle qu'il la chante. Comme je l'ai expliqué ailleurs ${ }^{24}$, l'entomologiste poète adopte une conception encore marquée par le catastrophisme de Cuvier et ses "révolutions du globe ${ }^{25}$, qui datent pourtant de plus de soixante ans. Cuvier, qui demeure la grande référence scientifique nationale, est nommé par trois fois ${ }^{26}$. Sa figure domine le poème et le modèle d'une façon déterminante : la conception en diachronie de la biologie qu'illustre Antediluviana repose davantage sur la révolution que sur l'évolution. Sa poétique en elle-même s'en trouve influencée. D'abord, Cotty rêve chacun de ces animaux comme s'il les voyait vivants, reconstituant la silhouette de tel ou tel monstre, comme Cuvier recomposait un être entier d'un fragment d'os ${ }^{27}$. De très nombreux passages du poème s'appuient sur une rhétorique testimoniale ${ }^{28}$ qui cherche à faire voir l'animal décrit par un souci renforcé d'enargeia: les verbes de perception se multiplient parfois de façon plaisante (« Mais qu'entends-je? que vois-je [...] ? », p. 3). Suivant la leçon cuviériste, les vers de Cotty déploient également une poétique de l'aposiopèse, ménageant de nombreux silences. Dans ces silences, les animaux se succèdent ("Au Reptile succède en grand le Mammifère » [v. 169]), surgissent les uns après les autres sans lien apparent. Ce cortège d'animaux qui défilent chronologiquement sous la plume du versificateur renvoie tout aussi bien au catastrophisme qu'à la muséographie séquentielle.

Toutefois, cette queue de comète du cuviérisme, en France, est marquée par une hyperréaction provoquée par la montée des thèses évolutionnistes. Ces cuviéristes tardifs, auxquels peut s'apparenter Cotty dans une certaine mesure, rajoutent une cause finale à la succession sans transition imaginée par le naturaliste (l'homme est le courronnement de l'évolution). Cuvier est ainsi trahi, et une nouvelle pensée de la biologie en diachronie voit le jour. Le traitement qu'en fait Cotty est complexe, puisqu'outre la conception cuviériste biaisée par cette approche téléologique, il y 
ajoute le souci tout positiviste de l'impossibilité de toucher jamais du doigt les causes premières et finales. L'humilité positiviste rejoint chez lui l'humilité chrétienne, comme chez Figuier, au point que l'aveu de non savoir devient un topos récurrent dans son poème.

Pourtant, il y a bien une certaine pensée de l'évolution dans cette pièce, mais elle emprunte peu au transformisme ou à l'évolutionnisme. En fin de texte, le biologiste amateur dévoile son point de vue : il relève plutôt d'une forme de création continuée, héritée probablement de Descartes. Cette pensée n'est pas si éloignée de celle d'Albert Gaudry (dont il cite d'ailleurs le nom dans son poème), qui défendait une forme de transformisme orchestré par Dieu ${ }^{29}$. Ainsi, le poème expose les animaux fossiles comme de simples essais, des esquisses sur lesquels se serait exercé Dieu avant de créer l'homme parfait. Le poème s'ouvre ainsi sur l'« ébauche » et l'«embryon » que constituent les premiers êtres pour se clore de manière héroïque sur la description et l'évolution à travers les âges de «l'œuvre culminante » que représente l'homme. S'il y a une évolution dans ce poème, elle est associée à une forme de rature, d'épanorthose biologique pratiquée de manière répétée par Dieu dans le temps long de l'histoire de la vie. L'animal fossile est une étude préparatoire. Cette conception n'est pas si originale, puisqu'elle est elle aussi reprise à Figuier :

[Le genre humain] venait d'où était venu le premier brin d'herbe qui apparut sur les roches brûlantes des mers siluriennes; d'où étaient venues les différentes races d'animaux qui se sont remplacées sur le globe, en s'élevant sans cesse dans l'échelle de la perfection. Il émanait de la volonté suprême de l'Auteur des mondes qui composent l'univers. ${ }^{30}$

18 Si ce poème mérite d'être lu, c'est parce qu'il témoigne des grands questionnements de son époque. Il met en scène de manière répétée les débats biologiques majeurs d'alors, en les citant précisément et en prenant ouvertement parti. Ce faisant il rend compte de la complexité de la science biologique en recherche d'assises stables au moment où l'évolutionnisme menace de chambouler la conception de la vie et de la place de l'homme dans le cosmos. Le texte se contemple également comme objet culturel complexe et n'allant pas de soi, en énonçant l'incongruité en même temps que la nécessité de la survie et de l'évolution d'une forme de poésie scientifique.

19 J'ai utilisé pour établir le texte le fascicule conservé à la Bibliothèque nationale de France sous la côte YE-40920, le seul disponible à ma connaissance, mais me suis également servi du texte identique paru dans la Revue de la société littéraire, historique et archéologique de l'Ain, numérisé sur Gallica. Le fascicule étant difficile à consulter, mais constituant jusqu'à présent l'édition de référence du texte (la dernière parue du vivant de l'auteur), j'ai indiqué entre crochets les numéros de pages. J'ai choisi de conserver la ponctuation quelque peu baroque de Cotty, qui, pour surprenante, n'en contient pas moins des effets de rythmes et de scansions éloquents, ainsi que quelques orthographes archaïsantes et coquilles que j'indique par le biais des notes.

\section{Antediluviana, poème géologique par Ernest Cotty}


Chant premier

Non, je ne prétends pas, des Temps préhistoriques

Dévoiler, dans ces chants, les arcanes féeriques,

À peine soulever un des coins du rideau

Qui nous montre, avec ordre, un merveilleux tableau :

5. Et la Flore et la Faune antédiluviennes,

Bien plus qu'on ne le croit, grandes magiciennes !...

Serais-je donc assez maître de mon sujet,

Pour en tenter l'abord, de plein pied ${ }^{33}$, d'un seul jet,

Sans préalable étude, avec inconscience ?...

10. Oh! ce n'est pas ainsi qu'on traite la science !

- Quel est donc cet intrus, dirait-on justement,

Qui franchit ce domaine aussi légèrement?...

La grandeur qui s'attache à la Géologie,

Comme à sa fille, la Paléontologie,

15 S'exprime en trop grands mots pour des alexandrins !...

- C'est vrai ; mais, puisqu'ils font route sur ces Terrains,

Il convient, même en vers, pour le sujet qu'on traite,

D'oser être technique,... ou de faire retraite.

[2] Époque primitive

Je laisse de côté l'épais brouillard gazeux,

20 Montrant notre Planète à l'état nébuleux,

Et, comme une âme en peine, errante dans l'espace,

- D'après la théorie émise par Laplace ${ }^{34}$,-

Puis, concentrant ses gaz et se consolidant,

Se faire bien petite au fond du Firmament.

25 Que dirais-je, d'ailleurs, de cette nuit lointaine?

La meilleure hypothèse est peut-être incertaine...

Je ne saurais donc pas, des vieux rocs primitifs

Exhumer en détail les Schistes collectifs;

Ni suivre, en ses Filons, la Matière éruptive

30 Qui monte à la Surface, en sa force éjective ;

Ni des Granits centraux, dans un sens arbitral,

Épiloguer à faux sur le Grain minéral...

Le peu que j'en dirais, pouvant être équivoque,

Je quitte, en son Chaos, la Terre, à cette Époque !

$$
\text { Époque de transition }
$$

35. Passons vite, de même, avec intention ${ }^{35}$,

Sur les autres Terrains, dits de Transition,

Où vont, s'arborisant, des Prêles, des Fougères,

Les vieux troncs que le temps a fait carbonifères ${ }^{36}$;

Premiers Âges du Monde, où le Règne animal

40 Cède le pas encore au Règne végétal...

Pour ne pas compliquer par trop le Catalogue

De ma muse hardie, et pseudo-géologue,

Je ne veux distinguer ni Sols siluriens,

Ni devoniens ${ }^{37}$, ni houillers, ni permiens ;

45 Où l'on ne voit, hélas! dans les Mers, apparaître

Qu'ébauches, qu'embryons d'existences à naître :

Trilobites hideux, parents des Crustacés,

Zoophytes, Poissons, à grand'peine classés, 
Encrines, entr'ouvrant ou fermant leurs calices, Et font des collecteurs les plus chères délices!

- Mais des Plantes d'alors les végétations

Ont déjà mille attraits pour leurs proportions :

Les Sigillaires, les gracieux Calamites,

Les Lépidodendrons, les Astérophyllites!

$$
\text { [3] Époque secondaire }
$$

I.

55. Après un temps fort long de siècles révolus, L'aspect se modifie... Alors, on ne voit plus Que Reptiles hargneux, puissants Maîtres du Globe, Dont plus d'un, dans la brume, aux savants se dérobe... C'est alors que surgit du sol, en abandon,

60 Un monstre étrange et lourd, le Labyrinthodon ;

Puis vient un Crocodile, appelé Nothosaure, Qui, sur les rocs visqueux, d'Encrines se restaure ; $\mathrm{Au}$ Terrain triasique ils remontent tous deux, Et traînent, répulsifs, leurs instincts hasardeux...

II.

65. À l'endroit où sera Paris, séjourne encore L'Océan qui du nom du Jura se décore ; Il recèle en ses eaux ces Mollusques éteints, Par les doctes chercheurs patiemment dépeints Et reconstitués ;... ces spires d'Ammonites,

70. Ces longs cônes pierreux, restants de Bélemnites ${ }^{38} ! \ldots$

- Mais, qu'entends-je ? que vois-je, au sein des vastes mers?

De terribles combats à mes yeux sont offerts :

Un Saurien marin, l'énorme Ichthyosaure,

Est aux prises avec l'affreux Plésiosaure ;

Ce dernier courbe en arc son long cou de serpent

Contre l'antagoniste, ,... à sa rage échappant !...

Sur une Cycadée, un fantasque Reptile,

Chauve-Souris, par l'aile ${ }^{39}$, est le Ptérodactyle, Qui, fondant sur sa proie, en un vol ébauché, Offre à son estomac un maigre débouché...

- Quel est cet hiéroglyphe, accusé sur le sable?

Du Ramphorynque ${ }^{40}$ c'est la trace irrécusable ;

Aîlé, comme l'est son précédent compagnon, Cette calligraphie est due à son moignon D'aîle, ainsi qu'à sa patte et sa traînante queue. Cette piste devait, pendant un quart de lieue, Trahir, et sa présence, et les pièges sournois Que, fourbe, il méditait dans l'ombre, en tapinois !... [4] Faut-il dépeindre aussi le vif Téléosaure,

90 Caïman cuirassé ?... - Pauvre Clémence Isaure ${ }^{41}$, Si ces vers étonnants lui passaient sous les yeux, Quel langage aujourd'hui parle-t-on, justes dieux! S'écrierait-elle ?... - Eh bien ! ici mon axiôme Est, que, selon le cas on change d'idiôme!

95 Quand de Géologie on veut causer un peu, On ne peut vraiment pas rêver fleurs et ciel bleu !... - L'Hyléosaure enfin, farouche, fantastique, Au dos de Hérisson, Être apocalyptique, (Comme en traçait Callot ${ }^{42}$ ), ne peut être oublié,

100 Bien qu'on ne le connaisse encore qu'à moitié...

- Pour détourner nos yeux de tant d'horribles Bêtes, 
Enfin vole un Oiseau, dans les airs tout en fêtes ;

C'est le premier !... Son nom c'est l'Archeopterix,...

Nom assez mal sonnant ${ }^{43}$, pour un Oiseau-Phénix !...

105 - Voilà le court bilan du Terrain jurassique.

J'ai fait tous mes efforts pour être véridique ;

On n'obtient pas toujours, en vers, ce que l'on veut...

Il faut être indulgent pour qui fait ce qu'il peut.

III.

Mais nous voyons s'ouvrir une autre Période,

110 C'est celle de la Craie; elle aussi s'inféode

Au Terrain secondaire... Ici nous allons voir

Défiler, devant nous, comme dans un miroir,

Auprès de vrais Palmiers, à la tige élégante,

D'autres monstres, pour nous, de forme extravagante :

115 L'Iguanodon féroce, et cherchant sourdement

À son rude ennemi querelle d'Allemand,

Peu soucieux du dieu que bénit Épidaure ${ }^{44}$,

Se rue, à belles dents, sur un Mégalosaure !...

Plus loin, on voit ramper un autre Saurien,

120 Dont l'histoire s'embrouille (un vrai nœud gordien)!

Ses fossiles débris, sur les bords de la Meuse,

En Hollande, ont été trouvés ; miraculeuse

Découverte, qui mit maint savant à quia...

Mais, pas moins, Maëstricht, lors s'en glorifia!

125 C'est le Grand-Animal du nom de cette ville;

Cru, tour à tour, Poisson, Baleine ou Crocodile,

[5] Il devint à la fin, par l'immortel Cuvier

Lézard de la Meuse, ou - pour l'inventorier, -

Mosasaure... O destin !... par le droit de conquête,

130 C'est Paris aujourd'hui qui possède sa Tête ${ }^{45}$ !...

Mais, dans mon rêve, moi, j'en voyais de vivants,

Dans la mer Crétacée ; ils narguaient les savants ;...

Et près d'eux j'admirais : Pernes, Térébratules,

Coquilles en volutes, en cercles, en spatules,

135 Mollusques folâtrant aux profondeurs des eaux,

Dans leurs maisons de nacre, adorables tableaux !

Époque tertiaire

I.

Remontons maintenant aux Couches éocènes,

Et nous verrons après les Terres miocènes ;

Car je désire, en hâte, à grands traits retracer

140 Cette Faune d'alors, qu'il ne faut qu'esquisser.

À cette Époque, on voit de puissants Pachydermes,

Mammiferes nombreux, aux rudes épidermes,

Broutant paisiblement: Palaeothérions,

Xiphodons au long col, Anoplothérions ;

145 Ils paissent en commun, dans le fond des vallées,

Les hautes herbes, près des lacs accumulées...

Louons du Créateur la divine bonté :

Car le Monde a déjà moins de férocité !

- La Flore tertiaire aussi se développe :

150 Les fruits du Banksia percent leur enveloppe ;

Les Arbres d'aujourd'hui, Chênes, Charmes, Ormeaux ${ }^{46}$,

Près de Palmiers, de Pins, confondent leurs rameaux,

Et l'Oiseau-de-Montmartre est perché sur leurs branches !...

- Je ne veux pas sombrer parmi les avalanches 
155 De tant d'Êtres divers : Tortue ou Trionyx,

Ni Vespertilio dit Parisiensis;

Ni me perdre en suivant les éocènes plages,

Pour scruter leur richesse, en fait de coquillages,

Terrestres ou marins: Physe, Helix, Cyproea,

160 Cyclostome, Cassis, Cardite,... et coetera!

Car leur liste, prenant un air de litanie ${ }^{47}$,

Pourrait rimer alors avec métromanie !...

[6] II.

La Faune miocène, et riche en habitants,

Dans la Faune actuelle a des représentants :

165 L'Éléphant, le Cheval, le Chat, l'Ours et le Phoque,

Et bien d'autres encore, animent cette Époque ;

Sans parler de tous ceux qu'on voit, - Dieu sait combien!

Dans la mer, avec leur manteau conchylien,

Au Reptile succède en grand le Mammifere ;

170 Et d'abord, à bon droit, il faut que je confère

La paille de la taille au Dinotherium,

- (Morse-Taupe-Tapir), que le critérium

De la science, après bien des doutes confine

Vers les types qu'a fait la Race éléphantine ${ }^{48}$. -

175 Des Animaux terriens c'est le plus colossal !... Ses Défenses, allant dans le sens vertical,

À peu près dans la courbe où sont celles du Morse,

Fouillaient le sol avec une incroyable force...

- Quel est ce Proboscide, aux abords de l'Ohio?

180 Il ne peut pas longtemps garder l'incognito ;

Sa carrure s'oppose aux erreurs, sur son compte...

C'est, à n'en pas douter, le fameux Mastodonte,

Analogue à celui qu'un sieur Mazuyerus

Produisit sous le nom de Rex Teutobocchus ;

185 Ce docteur Mazuyer, jouant à qui perd gagne,

À prix d'argent, fit voir, en France, en Allemagne, Les os de l'Éléphant... Louis-Treize, étonné,

Admira ce faux roi, venant du Dauphiné ${ }^{49}$ !...

- Mais un Singe, là-haut, nous fait force grimaces ;

190 C'est le Mésopithèque, ami des Calebasses

Des Noix du Cocotier, qu'il brise en les jetant

Avec dextérité, contre un sol résistant.

III.

Pénétrons maintenant dans les Temps pliocènes,

Où de Commotions s'incrustèrent les scènes,

195 Où la Terre trembla, jusqu'en ses fondements,

Par des Éruptions et des Effondrements ;

Par des Soulèvements de Groupes de Montagnes,

De Volcans, déchaînant l'enfer sur les campagnes !...

[7] Et nous verrons, plus tard, les Flots neptuniens

200 Ne le céder en rien aux Feux plutoniens !...

- La place où git Paris, - de Neptune héritière, -

Se montre hors des eaux de la Mer tertiaire, -

Et Marseille, à son tour, de nos bords provençaux

Pourra naître,... et, de même, en Gascogne, Bordeaux...

205 Mais leur place sera bien longtemps nue encore !...

La Nature nouvelle, aux sols venant d'éclore,

Pour habitants n'aura, dans ces Temps ténébreux,

Que tous ces Animaux, dont les débris pierreux 
Ont des échantillons aux Buttes de Montmartre...

Un blanc linceul de chaux les revêt de son tartre!

- La Terre, à cette Époque, est riche en Végétaux ;

Sa Flore plantureuse envahit nos coteaux...

Mais, dans nos régions, les Palmiers miocènes

Alors ont disparu des botaniques scènes,

215 En laissant subsister Noyers et Peupliers,

Et Platanes ombreux, qui nous sont familiers ;

Puis cent autres, ici ne pouvant trouver place,

Pour ménager le temps aussi bien que l'espace ;

Similaires produits des Arbres de ce jour,

Des pays tempérés décorant le séjour...

- Des Animaux nouveaux, d'un type hétérogène,

Caractérisent bien la Coupe pliocène :

D'abord, clopin-clopant, on voit le Glyptodon

Cheminer lentement, près du Schistopleuron;

225 Herbivores tous deux, vêtus de Carapaces,

Mammiferes comptant dans les tatouses Races ${ }^{50}$,

Bien qu'avec la Tortue, au tout premier abord,

Leur galbe extérieur ait beaucoup de rapport..

- Mais que vois-je ?... un colosse impossible, équivoque,

230 Velu, lourd et massif, d'un aspect fort baroque, Sa taille presque atteint celle d'un Éléphant...

Il a, malgré cela, l'air assez bon enfant ;

Mais l'ampleur de ses pieds est vraiment ridicule;

Aucun autre Animal n'en a de ce module.

235 Du mangeur de verdure, au long groin de Tapir, La lugubre ossature, à Madrid, fait frémir !...

[8] Il vient du Paraguay; la science le nomme

Le Megatherium ${ }^{51}$... Très-remarquable, en somme !

- Pour faire un beau pendant au Megatherium,

240 Quel autre meilleur choix qu'un Sivatherium?

Le Sivatherium est un Cerf gigantesque,

Haut comme son voisin, - ce Paresseux grotesque ;-

Son aspect est étrange, avec ses quatre Bois ;

Jamais ne l'a troublé le cor, au fond des bois ${ }^{52}$...

245 Hippopotames, Bœufs, Chameaux, troupeaux sauvages, Animent de ces lieux les verdoyants rivages !

- Dans le Bloc que voici, c'est l'apocryphe Homo Diluvii testis; que Scheuchzer, en un mot, Désignait sous ce nom... Toute une polémique

250 Alors s'épanouit, dogmatico-comique :

En mil sept cent vingt-cinq, mein Herr doctor Scheuchzer, En Suisse découvrit (bon prétexte à rêver),

Cette merveille !... - Hélas ! qui mit fin à l'esclandre, Prouva que ce n'était rien qu'une Salamandre,

255 (Mais une Salamandre hors de proportions, D'un Crocodile ayant bien les dimensions)?

Ce fut encore ${ }^{53}$ Cuvier, le grand naturaliste, D'un prodige augmentant sa glorieuse liste !... Lors, ce Batracien, quittant son pilori, Prit le nom régulier d'Andrias Scheuchzeri ${ }^{54}$.

[9] Chant second

Époque quaternaire

I. 
Sur le sol moins ancien des Terrains quaternaires, Nous dirigeons enfin nos pas imaginaires ;

Cette Époque où le Roi de la Création

Sur la Terre fera son apparition;...

Du Divin Créateur cette œuvre culminante,

Portant du sceau sacré la marque rayonnante !...

Bien des siècles encor pourtant s'écouleront,

Avant que l'Homme, ceint d'un diadème au front, Prenne, majestueux, le sceptre de ce Monde !...

270 Avant de l'y créer, ô sagesse profonde !

Dieu veut de ce séjour adoucir les rigueurs,

L'expurger des Tyrans ${ }^{55}$ des Temps antérieurs,

De ces Êtres à fuir, dont les Faunes éteintes

Portent fidèlement les terribles empreintes !

275 Mais plus d'un Cataclysme, en ces sombres moments, Doit encor renverser l'ordre des Éléments !..

Avant de contempler ces grandes Catastrophes,

- Qui, de nos Temps présents sont presque limitrophes, -

Signalons, à la hâte, aux bords des nappes d'eaux,

280 À l'ombre des forêts, des Animaux nouveaux :

D'abord un Éléphant, aux Défenses arquées,

Dont les courbures sont même assez compliquées,

Le Mammouth (Elephas primigenius) ; son

Énorme et blanc squelette au cœur donne un frisson!

285 Du Proboscidien la forme colossale

Se voit à Pétersbourg, dans une vaste salle

Du Muséum ${ }^{56}$... D'abord, avec chairs, poils touffus,

Avec soin, fut extrait cet antique reclus,

Des glaçons disloqués de la Mer glaciale...

290 La science y conquit sa palme triomphale !..

Le Mammouth, en partage, eut l'honneur souverain

D'être du Mastodonte un peu contemporain.

[10] - Un fort Rhinocéros, à toison luxueuse,

À deux Cornes, fut pris (relique ${ }^{57}$ fastueuse

295 Pour la Géologie !) aux bords sibériens,

Dont les sables glacés étaient les gardiens ;

Il orne aussi le beau Cabinet moscovite...

C'est au Germain Pallas qu'en revient le mérite ${ }^{58}$.

- Faut-il parler aussi de l'Ursus speloeus,

300 (Ours fort mal léché), dont la Race ne vit plus?..

Du Felis speleoa, ce Tigre de caverne?..

De l'Hyène cauteleuse, à bravoure assez terne ?..

Du Cerf hybernien (Cervus megaceros),

Autrement encorné que le Rhinoceros?

305 - Pour finir avec cette âpre Zoologie,

Un seul mot, ayant trait à l'Ornithologie :

Car, depuis qu'a paru l'Oiseau Solen-Hofen,

La famille empennée a peuplé son Éden ${ }^{59} .$.

Ici donc, vient l'Oiseau, de grandeur gigantesque,

310 Qui vécut en des lieux qui sont inconnus, presque :

La Nouvelle-Zélande a vu ce Dinornis,

Qui nous légua ses os... - Quant à l'Epiornis,

Madagascar, hélas ! n'a pu donner encore

Que ses Cufs, cinq fois comme ceux qu'élabore ${ }^{60}$

315 L'Autruche,... qui, sans peur dans le faux de tomber, Pour la taille, ont pourtant le droit de s'exhiber ${ }^{61}$ ! 
II.

Après avoir percé la longue Nuit des Âges,

Nous allons retracer les sinistres images

Des Temps diluviens, - plus rapprochés de nous, -

Où vont se déchaîner les Ondes en courroux...

Une preuve évidente, au sol s'en développe,

Confirmant, à nos yeux, les Déluges d'Europe,

Ces envahissements des indomptables Eaux,

Effets de la Fournaise aux puissants Soupiraux !..

325 - Le Démon qui préside à ces noirs Cataclysmes,

Des Feux intérieurs règle les Vulcanismes ;

Ces Laves, ces Brasiers, toujours incandescents,

Des Fluides gazeux, sans cesse menaçants,

[11] Activent, bruyamment, la rage épouvantable !..

330 Notre Écorce terrestre, au Fléau redoutable,

Ne pouvant opposer, en sa faible épaisseur,

Nulle digue aux fureurs du Flot envahisseur,

- Flot de torrents de feu, - se déchire et s'entr'ouvre,

Pour le laisser passer !.. Mais bientôt se découvre

335 La naissance subite, au sein des Océans,

De Granits éjectés par les Gouffres béants !..

Des Monts altiers soudain se dressent dans les plaines,

Soudant à d'autres Monts leurs éruptives Chaînes !..

Alors la Mer déborde, enveloppant partout

340 Le terrain affaissé, qu'elle inonde et dissout !..

Produits amoncelés des Vapeurs du Cratère,

Des Nuages épais, surchargeant l'Atmosphère,

Se résolvent en Pluie, en Trombes ; - amas d'eau

S'unissant à la Mer,... qui cherche son niveau !

345 C'est en ces Temps, témoins de terribles spectacles,

Que cette Onde sans freins, surmontant mille obstacles,

Portant partout la mort, la désolation,

Nous montre, après le mal, sa dévastation!

- Or, le premier Déluge, éclatant sur les terres

350 Du continent d'Europe, - et dont les caractères

Sont, par le Géologue, au vif déterminés, -

C'est celui qui sévit, en bonds désordonnés,

(Par les Monts soulevés de la Scandinavie),

Sur les Pays Germains et sur la Moscovie !..

$355-$ C'est ainsi que l'Islande, en son polaire exil,

Vit émerger des Flots son rigide profil ;

Que, pour dompter l'effet des infernales sapes,

Vainement ses Volcans font jouer leurs soupapes!

Le deuxième Déluge, en Europe engendré,

360 Par le Soulèvement alpestre est consacré...

- Dieu seul connaît le but, en ses suprêmes actes,

Des lois qui font s'ouvrir, bondir ces Cataractes

Sur notre triste Monde, en tous ${ }^{62}$ temps subissant

Tant de calamités ,... - à les vaincre impuissant !.. -

365 Mais tout, du Créateur, avec respect s'impose :

Si nous voyons l'effet, Lui seul en sait la cause ${ }^{63}$ !

[12] III.

Mais il nous faut encor des Révolutions

Du Globe, élucider d'autres solutions ${ }^{64}$ :

- Essayons de dépeindre, en suivant sa filière,

370 Aux régions du nord, l'Époque glaciaire, 
Ce phénomène aussi terrible que soudain, Avant de voir enfin naître le Genre humain !.. L'Europe, presque entière, aux Glaces condamnées, Du Pôle nord jusqu'à la Méditerranée, D'un Refroidissement a subi les rigueurs ; Des climats exposés à de chaudes ardeurs Furent sous le coup d'une implacable Gelée ; Avec son manteau blanc, la Terre désolée Devint un froid linceul, qui, brisant le ressort De la vie, accomplit sa grande œuvre de mort !.. Des Animaux géants (surprenants amalgames), Victimes, tout à coup, des inflexibles drames, Nous ont laissé, complets, dans des Blocs de Glaçons, - Sarcophages pour eux, aussi bien qu'étançons, -

385 Leurs cadavres !.. - Hélas! merveilles exposées Avec soin, aujourd'hui, dans nos riches Musées !.. De ces faits, seule encor la constatation Est produite, mais non leur explication... On ne peut que former ici des hypothèses ;

390 D'une énigme, pour nous, Dieu seul sait les synthèses !.. Qui, nous dit, qu'en ces Temps, le Divin Créateur, De l'Homme, par avance, ardent libérateur, Avant de le placer dans son royal Domaine N'ait voulu protéger sa Créature humaine,

395 Pour elle atténuant l'écueil de bien des maux, Par la mise au néant d'un surcroît d'Animaux? Comment prouver l'effet de pareils phénomènes, De Glaciers, étendant, sur tant d'immenses plaines, Leurs ravages, qui n'ont nulle trace aujourd'hui?

400 La science, ici, va nous prêter son appui : Pour comprendre les faits des Temps géologiques, De nos jours il faut voir les faits analogiques, [13] Qui, pour être mesquins, en leur infimité, N'en constatent pas moins une réalité...

405 Ce qu'il reste, à présent, des Glaciers d'Helvétie, Recouvrait autrefois une superficie Qui s'étendait au loin, depuis les monts alpins, Presque jusqu'aux vallons, base des Apennins !.. De siècle en siècle, alors il nous faut en déduire

410 Que l'on voit ces Glaciers, sans fin se circonscrire, N'être que l'ombre enfin des Glaciers d'autrefois ; Mais imposants toujours, possédant, à bons droits, Le séduisant appas d'attirer les touristes, Les savants, du vrai beau justes apologistes ${ }^{65}$;

415 Chacun à sa manière, examinant l'effet Que sur lui sait produire un semblable sujet !.. Ces Neiges, ces Glaçons, fuyant leurs crêtes blanches, Ces Rochers, entraînés, aux chocs des Avalanches, Expliquent, en petit, les émouvants tableaux

420 Dont le Temps glaciaire engendra les fléaux. Ils démontrent comment ces grands Blocs erratiques Des Glaciers de Norvége ${ }^{66}$, - et que les flots baltiques, Dans leur long glissement, n'ont pu même arrêter, Au centre de l'Europe, en venant échouer,

425 De la Géologie ont comblé les surprises !.. Mais le fait est patent pour ses doctes assises ! 
Seulement, nul n'a pu nous dévoiler comment, Par quelle cause eut lieu ce fatal Glacement?

- Dans la science, hélas! (comme Figuier l'exprime,

430 En un livre érudit ${ }^{67}$, dont s'inspira ma rime) :

Il faut oser, sans honte, en y portant ses pas,

Parfois, fort humblement, dire : Je ne sais pas!

IV.

Humains, inclinez-vous !.. car voici cette aurore,

Qu'en ses divins décrets, pour vous, Dieu fit éclore !...

435 Voici le jour béni de l'apparition

Du légitime Roi de la Création!

Sur les bords protégés du Tigre et de l'Euphrate,

Est né le premier Homme !.. Il devient l'autocrate

[14] De la Terre, qui va se soumettre à ses lois

440 Car l'Éternel lui-même a consacré ses droits ;

Tutélaires appuis, nobles investitures,

Grands legs, qu'ont confirmé ${ }^{68}$ les Saintes-Écritures,

Que Moïse inspiré, sage initiateur,

- Ce prophète sublime, et de Dieu traducteur, -

445 Nous transmit; car Dieu dit : "Que l'Homme, à notre image,

Du don de la raison ait le sceptre en partage ;

Qu'aux Bêtes il commande, aux Poissons de la mer,

Comme aux Oiseaux du ciel qui voltigent dans l'air ${ }^{69}$ ! »

Dans ces lieux enchanteurs, que la Bible dénomme,

450 Sous le souffle divin a donc apparu l'Homme !..

Il est bien exposé, dans ses commencements ;

Car il lui faut lutter contre les Éléments,

Contre mille dangers, lui, faible et sans défense...

Mais il deviendra fort : Il a l'intelligence !

455 Il se revêtira, se fera des engins

Qui devront assurer ses glorieux destins...

Et tous les Animaux, redoutant sa puissance,

Reconnaîtrons alors sa juste omnipotence !..

Soumis au joug vainqueur, leur imposant son frein,

De l'Homme ils sentiront le pouvoir souverain!

$\mathrm{V}$.

Multipliant bientôt son adamique Race,

Du Genre humain la Terre au loin porta la trace...

Mais du vieux Globe, hélas ! les Révolutions

N'ont point fermé leur ère aux dévastations :

465 D’un Déluge nouveau la rage frénétique

Soudain vint éclater, au centre asiatique !..

Le Monde s'agita, jusqu'en ses profondeurs ;

Tonnerres souterrains, Feux exterminateurs,

Font onduler le Sol, comme une mer ondule ;

D’un long Déchirement, effrayant préambule !..

Et la Fournaise éclate, et le Gouffre vomit

La Matière qui boût ${ }^{70}$ et brûle, dans son lit...

Ces Laves, s'échappant du grand Laboratoire,

Déversant au dehors leur bouillant exutoire,

475 [15] Plongeant, en même temps, la Terre dans la nuit, En plein jour, font douter si l'Astre du Jour luit !

Un Réseau montagneux, au midi du Caucase,

Soulève de ses Pics la volcanique Base ;

Et, des champs d'Arménie, en ce rude combat

Des Éléments, surgit le grand Mont-Ararat, 
Aux pitons recouverts de neiges éternelles !..

Du drame plein d'horreur les phases solennelles, Par l'écrivain sacré, dans d'émouvants récits, Ont gravé leurs tableaux, au fond de nos esprits... Et les traditions du Déluge biblique Ont conservé partout une trace authentique : Le Coran, les Védas, la Genèse en font foi, Donnant à la science un fraternel octroi... Car de Moïse alors, la sublime parole,

490 Sur « l'abîme des eaux, » n'est point une hyperbole ; Et ce qu'il nomme encor " cataracte du ciel, $»^{71}$ Le Mexique en offrit un effet partiel, Dans le siècle dernier : Notre époque en est juge !.. Or, l'Inondation de l'antique Déluge

495 Mosaïque, a produit tous les maux désastreux Qu'énumère la Bible, en versets douloureux : Les campagnes, partout, se trouvant inondées, Et les boueuses Eaux, «jusqu'à quinze coudées ${ }^{72}$ " $\mathrm{Au}$-dessus des Sommets dépassant la hauteur,

500 Aucun terrain n'offrit son habit protecteur Aux Humains, succombant dans ce naufrage immense !.. Et tous les Animaux, atterrés, en démence, Malgré le triste essai d'un lamentable effort, Furent anéantis, par l'implacable sort !..

505 - C'est bien sur l'Ararat que Noë prit refuge, Dans son Arche bénie, échappant au Déluge...

- « Toute la terre alors fut cent cinquante jours « Sous les eaux ${ }^{73}$, - dit Noë,... Qu'il faut croire toujours !.. Mais la Sainte-Écriture et la Géologie,

510 (Qui paraissent n'avoir aucune analogie), Vont se trouver ici, ce semble, en désaccord... Désaccord apparent ; car, sans beaucoup d'effort, [16] En suivant les extraits d'un savant Géologue, - Qui se montre, à la fois, un profond philologue, -

515 Le mot « toute la terre, » à faux considéré Par la Vulgate, n'a que le sens figuré... Marcel de Serres dit, en sa Cosmogonie De Moïs $e^{74}$ : le terme haarets, par le génie De la langue hébraïque, a l'application

520 Fort souvent de Pays, de simple Région; L'auteur sacré n'a donc, par son noble langage, Fait d'autre allusion, dans sa sincère image, Qu'aux Pays fort restreints, et que peuplait alors L'Humanité naissante, éparse sur ces bords...

525 Pourtant, la catastrophe, en sa fougue ennemie, Au sol fertile où fut la Mésopotamie, A dû, non-seulement étendre son horreur, Mais se porter au loin, en égale fureur. - Ces fléaux dont la Terre a vu l'effervescence,

530 Ne les maudissons pas !.. car c'est la Providence Qui les a résolus, en ses desseins secrets... Il faut nous incliner devant tous ses arrêts!

VI.

Mais j'arrive à la fin de ce trop court poème ;

Car je n'ai qu'effleuré le vaste et beau problème Dont la Géologie exhume les grandeurs! 
- Honneur donc, à jamais, à ces ardents chercheurs Dont les puissants travaux, de la science humaine, Pour tous, ont élargi le glorieux domaine!

Gloire aux Cuvier, Brongniart, Beaumont, Hébert, Vibray, Verneuil, Lartet, Gaudry, Collomb, Buteux, Mercey ${ }^{75}$ !..

J'interromps à regret le cours de cette liste,...

(Bien que l'on doive, en tout, être un peu formaliste).

Chez les Anglais, pourtant, citons d'autres savants :

Lyell, Flower, Prestwich, Falconer, Busk, Evans ${ }^{76}$,

545 Qui prirent part active aux Fouilles d'Abbeville, Lorsque Boucher de Perthe $e^{77}$, orgueil de cette ville, Dans le Diluvium, où git Moulin-Quignon,

Mit, en Géologie, un triomphal jalon !..

[17] - Nous allâmes, plus tard, en grande Compagnie,

550 (De la belle cité, reine de Picardie),

Rendre au docte vieillard, Boucher de Crèvecœur

De Perthes, un hommage ;... et du noble vainqueur

Admirer le Musée (où vit l'Âge de pierre),

Posthume nécropole, auguste cimetière,

555 Dont nos vieux Ascendants, - ô dura lex, sed lex ! -

Taillèrent autrefois les Haches en Silex !

C'est en soixante-trois, et le jour vingt-huitième

De Mars, que notre illustre hôte (bonheur suprême !)

Découvrit son Prodige antédiluvien,

560 Qui mit tout en émoi le monde européen ;

Ce trophée imposant d'une Mâchoire humaine

Fossile, reposait sur la crayeuse arêne ${ }^{78}$

De Quignon !. Bien longtemps, on le lui contesta !..

Enfin la vérité, trop tardive, éclata :

565 On couvrit de lauriers le front du Géologue;

Et la science admit, dans son grand catalogue,

(Livre-d'Or du savoir), par acclamations,

Ce vieux Vestige humain des générations !

- Comme on fait pour les Saints, dans mainte basilique,

570 Grandiose débris, vénérable relique,

On devrait te garder comme un pieux trésor,

Sur un lit de velours, dans une châsse d'or!

VII.

Hélas! bornons ici nos trop vives louanges !..

Dans notre Race humaine, il est bien des mélanges,

575 Et l'ivraie, ici-bas, s'amalgame au bon grain ${ }^{79}$..

L'Homme, à ses passions en n'imposant nul frein,

Méconnaît sa raison, dont Dieu l'a fait l'arbitre ;

Roi de ce Monde, alors il n'en a que le titre...

S'il veut du Créateur mériter les bienfaits,

580 Il lui faut rester sourd à ses instincts mauvais,

Bannir, à tout jamais, les guerres homicides,

Ces forfaits insensés, - tristes éphémérides ! -

Qui font se déchirer tous les peuples entre eux,

$\mathrm{Au}$ lieu de vivre en paix, sages, libres, heureux !..

585 [18] S'il en était ainsi, nous irions en dérive.....

Il est temps, ô mon Dieu, que votre Règne arrive !..

Que votre Volonté soit faite 80 !.. Et vos Enfants,

Au céleste séjour entreront triomphants! 


\section{NOTES}

1. Entomologie: Observations à propos du Bombyx Cynthia; relation de quelques chasses de coléoptères rares d'Algérie, Amiens, Lemer aîné, 1867 et Particularités curieuses sur différents reptiles, insectes et crustacés d'Algérie, Amiens, impr. De Caillaux, 1869. Ces deux textes ont d'abord paru dans les Publications de la Société linnéenne du nord de la France.

2. Description du Musée d'histoire naturelle et du Jardin botanique et zoologique de Tours, extrait des Publications de la Société linnéenne du nord de la France, Amiens, impr. de Lenoël-Hérouart, 1871. Il y sera désormais fait référence sous la forme abrégée : Description du Musée...

3. L'Entomologie. Ode sur les coléoptères, Bourg, impr. de Comte-Milliet, 1874 (paru un an plus tôt dans la Revue de la société littéraire, historique et archéologique de l'Ain, $2^{\mathrm{e}}$ année, 1873, p. 380 à 383) et Antediluviana. Poème géologique, Bourg, impr. de Comte-Milliet, 1876 (paru un an plus tôt, en deux livraisons, dans la Revue de la société littéraire, historique et archéologique de l'Ain, 1875, 4 année, p. 281-288 et 336-345).

4. Dans la Revue de la société littéraire, historique et archéologique de l'Ain sont parues "Chant du matin », $1^{\mathrm{e}}$ année, 1872, p. 427-428, «Le Crabe et l'Écrevisse », fable imitée de La Fontaine, 1873, $2^{\mathrm{e}}$ année, p. 95, "Promenade en mer au clair de lune », 1873, $2^{\mathrm{e}}$ année, p. 319, « Ligugé », 1873, $3^{\mathrm{e}}$ année, p. 190-191, et « Le Porte-bonheur », 1875, $4^{\mathrm{e}}$ année, p. 64.

5. Louis Figuier, La Terre avant le déluge [1863], Paris, Hachette, 1866.

6. Edmond Emerich, La Création du globe terrestre. Poème géologique, Strasbourg, impr. de Vve. Berger-Levrault, 1860.

7. L'adjectif antediluvianus est très rare en latin. La base de données Database of Latin Dictionaries de Brepols ne le repère que dans un livre publié en Hongrie en 1802 : Nova memoria Hungarorum et Provincialium scriptis editis notorum, quam excitat Alexius Horányi. Pars I, Pestini, 1802, p. 200, cité par Antonius Bartal, Glossarium mediae et infimae latinitatis Regni Hungariae, Budapest, 1894. Il semblerait plutôt que le mot ait été forgé à partir de l'adjectif français antédiluvien attesté depuis le XVIII ${ }^{\mathrm{e}}$ siècle. Je remercie Jacques Elfassi pour ces informations.

8. Pour un développement sur l'usage du technolecte dans la poésie du second $\mathrm{xIX}^{\mathrm{e}}$ siècle, et notamment dans les poèmes de Cotty, voir mon article « Des coups de marteau dans la langue des dieux : mettre en vers le technolecte en un siècle positiviste », Un territoire en partage. Littérature et sciences au XIX $X^{e}$ siècle, Elsa Courant et Romain Enriquez (dir.), ouvrage disponible en ligne depuis le 18 janvier 2018 sur le site Épistémocritique. En ligne: http://epistemocritique.org/coups-demarteau-langue-dieux-mettre-vers-technolecte-siecle-positiviste/.

9. Vers 283-284.

10. Par exemple les vers 430 à 433 («Dans la science, hélas! [...] / Il faut oser, sans honte, en y portant ses pas, / Parfois, fort humblement, dire : Je ne sais pas!») sont une mise en vers du texte de Figuier : ce dernier renonce à donner une cause à la période glaciaire en concluant sur ces mots: «Dans les sciences il ne faut jamais craindre de dire : Je ne sais pas. ", La Terre avant le déluge, op. cit., p. 420.

11. "Le Crabe et l'Écrevisse ", Revue de la société littéraire, historique et archéologique de l'Ain, op. cit.

12. Description du Musée..., op. cit., p. 53-54.

13. Ibid.

14. J'indiquerai par le biais de notes les vers qui me semble le plus directement inspirés de la Description du Musée...

15. Ibid., p. 24. C'est Cotty qui souligne et met en gras.

16. Reste à déterminer si l'insertion du technolecte est également une conséquence logique des poétiques romantiques qui souhaitent ouvrir la porte du vers à tous les mots du langage, ou bien 
si elle est en contradiction avec ce même Romantisme qui revendique plutôt un accès au territoire versifié aux mots roturiers. Or rien n'est moins élitiste que la langue des savants.

17. Vers 81-85.

18. Le trimètre romantique a la particularité de présenter deux accentuations alternatives d'un même vers, puisqu'outre le rythme du trimètre, en $4 / 4 / 4$, la césure doit également être respectée (6+6). Par conséquent, il peut être modélisé comme suit : 4/(2+2)/4.

19. À titre de comparaison, Rimbaud vient d'achever son voyage poétique révolutionnaire et Ducasse a publié les Chants de Maldoror six ans auparavant. Ces deux œuvres renouvellent, chacune à leur manière, et de façon plus fondamentale que chez Cotty, le dialogue entre la poésie et la biologie.

20. L'Homme des champs, Paris, nouvelle édition, chez Levrault, Schoell et C $C^{\text {ie }}, 1805$, p. 107.

21. J'indique ces intertextes en note dans le texte.

22. Seul Pallas est cité (v. 298). Toutefois, il travaille pour Catherine II de Russie; ses découvertes ne sont pas mises au crédit de l'état germanique.

23. Haeckel est l'un des grands absents du poème de Cotty. Force est de constater, par ailleurs, que les poètes français traitant de sciences sont très rares à lui faire une place. Seul Richepin, reprend directement ses théories dans La Mer (1886), et encore le fait-il sans citer le biologiste allemand. La précision semble suggérer que son jugement n'est pas « scientifique ».

24. "Evolutionism and successivity in Antediluviana, Poème géologique by Ernest Cotty (1876)", Biological Time, Historical Time. Transfers and Transformations in 19th Century Literature, Niklas Bender et Gisèle Séginger (dir.), Leiden/Boston, Brill Rodopi, coll. » Faux Titre », 2018, p. 279-293.

25. Dans le texte, le mot révolution apparaît deux fois. Au vers 367 , il est souligné par l'effet de discordance qui le place sous accent rimique et le sépare brutalement de son complément du nom : "Mais il nous faut encor des Révolutions / Du Globe, élucider d'autres solutions »; il est également mis à la rime lors de sa seconde occurrence, au vers 463.

26. P. 5,8 et 16 .

27. C'est ici aussi à la poétique de La Fontaine, capable de faire apparaître d'un trait allusif et fragmenté une silhouette complète, que l'influence de la méthode cuviériste semble inviter Cotty. 28. Rhétorique que Cotty reprend également à Ovide dont les vers cités en exergue constituent un exemple.

29. Selon Gaudry (1827-1908), Dieu ne détruit pas les espèces mais les parfait au fil des siècles.

30. La Terre avant le déluge, Louis Figuier, op. cit., p. 421.

31. La Société entomologique de France, toujours active, a été fondée en 1832 par dix-huit entomologistes parisiens. C'est la plus ancienne société d'entomologie. La Société linnéenne du Nord de la France (aujourd'hui Société linnéenne Nord-Picardie) fut créée en 1838 à Abbeville sous l'impulsion de Casimir Picard, médecin et préhistorien ami de Jacques Boucher de Perthes. Elle est florissante dans les années 1860, et Boucher de Perthes fait partie de ses plus illustres membres.

32. Je traduis: "J'ai vu également des terres surgies des eaux / Et loin de la haute mer des coquilles marines répandues ».

33. Sic.

34. Le Français Pierre-Simon de Laplace (1749-1827) postule, selon l'hypothèse de la nébuleuse, que le système solaire était d'abord composé d'une masse gazeuse tournant autour d'un axe. Cette masse se refroidissant, des anneaux se sont formés et détachés, créant les planètes du système solaire. Cette hypothèse est encore en vigueur de nos jours. Elle est défendue en 1796 dans L'Exposition du système du monde.

35. Vers qui rappelle un passage de la Description du Musée... où l'auteur souligne également un silence : «Je saute avec préméditation les Perdrix et les Cailles », op. cit., p. 32.

36. Référence à la fossilisation. 
37. On attendrait «dévoniens ». Toutefois, l'absence d'accent peut s'expliquer par le fait que le mot, hérité de l'anglais, n'est qu'en voie de francisation. Il faut attendre 1878 pour le trouver dans le dictionnaire de l'Académie.

38. Cette rime facile est probablement inspirée d'une liste issue de la Description du Musée... : "quelques grandes Ammoniytes, des Bélemnites, des Encrinites», p.49. Il semble que cet homéotéleute accidentel ait plu à Cotty.

39. Écho plaisant à la fable de La Fontaine «La Chauve-Souris et les deux Belettes » ("Je suis oiseau ; voyez mes ailes»), qui interroge précisément la nature instable de la chauve-souris et conclut sur la nécessité d'adaptation. Cotty avait ces vers en tête, puisqu'il les cite précisément dans sa Description du Musée..., op. cit., p. 9.

40. On attendrait « Rhamphorhynque », c'est-à-dire littéralement « bec-mâchoire ».

41. Personnage légendaire de la fin du Moyen-Âge, Clémence Isaure est l'incarnation de la poésie, puisque, grâce à ses largesses, elle aurait fondé (ou restauré) les Jeux Floraux de Toulouse, concours de poésie qui existe encore. La position de son patronyme à la rime avec « Téléosaure » est une provocation plaisante en même temps qu'une illustration de l'union du poétique et du scientifique de la part du biologiste poète.

42. Dessinateur et graveur lorrain (Nancy, 1592-1635), Jacques Callot est devenu une figure prédominante pour les auteurs $\mathrm{du} \mathrm{XIX}^{\mathrm{e}}$ siècle se réclamant du grotesque, et notamment les romantiques. Aloysius Bertrand, parmi les premiers, met dès le sous-titre de Gaspard de la nuit (1842), ses pièces sous le patronage de Callot (Fantaisies à la manière de Rembrandt et de Callot). Hugo revendique également ce patronage esthétique, grimaçant et rieur (voir par exemple « Ce qui se passait aux Feuillantines vers 1813 ", dans Les Rayons et les ombres [1840]).

43. Expression héritée des réflexions faites à propos de l'aigle dans la Description du Musée... : " oiseau altier, imposant et superbe, en dépit de sa qualification mal sonnante, mais technique [Oiseau de proie ignoble] », op. cit., p. 18. Je souligne.

44. Ville de l'Antiquité grecques où se trouvait un important sanctuaire dédié à Asclépios, dieu de la médecine et de la guérison.

45. Le crâne de ce squelette fossile fut effectivement ramené à Paris après le siège de la ville de Maastricht par les troupes révolutionnaires françaises.

46. Exemple significatif d'engendrement phonétique de la liste. Le poème de Cotty en est riche.

47. Le mot est utilisé à propos d'une liste jugée elle-aussi trop longue, dans la Description du Musée... : « et d'autres dont je passe les noms pour n'en pas faire une litanie. », op. cit., p. 28. Je souligne.

48. Expression plaisante et néologique qui constitue un nouvel écho discret à La Fontaine (on peut penser, par exemple, à « la race escarbote », dans « L'Aigle et l'Escarbot »).

49. Teutobochus est un géant légendaire, fruit d'une supercherie, constitué à partir d'os de mastodonte (généalogie établie par Blainville en 1832) trouvés dans le Dauphiné en 1613 par un chirurgien de l'époque, Pierre Mazuyer. Son squelette fut présenté à la cour royale où il étonna fort le jeune Louis XIII. L'appartenance au règne humain de Teutobochus fut immédiatement sujette à débat.

50. Autre écho plaisant à La Fontaine.

51. Voir à son propos l'article de Cuvier, « Notice sur le Squelette d'une Très-grande Espèce du Quadrupède Inconnue Jusqu'à Présent, Trouvé au Paraguay, et Déposé au Cabinet d'Histoire naturelle de Madrid », Magasin Encyclopédique, vol. I, n³ 3, 1796, p. 303-310.

52. Référence plaisante au «Cor» de Vigny. Cet écho et les deux vers suivants esquissent une surprenante description d'un âge d'or animal préservé des appétits cynégétiques de l'homme.

53. Il faudrait, comme c'est le cas plus bas, adopter ici la licence poétique « encor». En l'état, le vers comporte une syllabe surnuméraire. 
54. En 1726, Johann Jakob Scheuchzer, médecin et naturaliste suisse connu surtout pour avoir interprété les fossiles comme des vestiges diluviens, découvrit à Oeningen, près du lac de Constance, un squelette fossile qu'il appela Homo diluvii testis («homme qui fut témoin du Déluge ») : il estimait qu'il s'agissait d'un homme noyé lors du déluge biblique. Il mesurait un mètre et pouvait ressembler à un enfant. Cuvier en 1812 établit qu'il s'agissait en réalité d'une espèce de salamandre.

55. Le choix de ce vocable permet une discrète lecture politique (républicaine) de ce poème qui défend une approche révolutionnaire de l'histoire du vivant.

56. Il s'agit du Mammouth d'Adams (Michael Friedrich Adams, 1780-1832), reconstitué en 1812 à Saint-Pétersbourg par Wilhelm Gottlieb Tilesius (1769-1857).

57. Le reste fossile sera à nouveau rapproché de la relique de saint à la toute fin du poème. C'est dire la valeur spirituelle que lui apporte Cotty.

58. Entre 1768 et 1874, Peter Simon Pallas (1741-1811), protégé de Catherine II de Russie, entreprit une expédition scientifique dans les provinces centrales de la Russie et en Sibérie. Il y récolte entre autres un nombre important de spécimens fossiles.

59. Effectivement, l'ornithologie est une nouvelle venue dans le champ de la paléontologie. En 1861 est découvert en Bavière le fossile de cet oiseau phénix que Darwin, dans une édition de L'Origine des espèces, considérait comme une forme de transition entre le reptile et l'oiseau.

60. Vers faux.

61. Ces vers sont la réécriture d'un passage de la Description du Musée... : « [...] il y a dans une autre armoire vitrée, consacrée à la paléontologie, une imitation, grandeur naturelle, de l'œuf d' Epyornis, cet oiseau fossile, colosse d'une époque antédiluvienne, trouvé à Madagascar ; lequel œuf est gros au moins six fois comme celui de l'Autruche du Sahara, pourtant d'une taille déjà fort respectable ", op. cit., p. 39.

62. Sic.

63. Réécriture du vers de Delille » Nous voyons les effets, Dieu seul connaît les causes ", Les Trois Règnes de la Nature, Paris, chez Giguet et Michaud, 1809, chant IV, p. 269.

64. Terme à prendre au sens vieilli de séparation (que l'on retrouve dans la solution de continuité).

65. Étonnante formule qui réunit dans le choc lexical la Bible et la science.

66. Sic.

67. Dans La Terre avant le déluge, op. cit., p. 420.

68. Sic.

69. Traduction en vers de Genèse, 1 : 26 .

70. Sic.

71. Genèse, $7: 11$.

72. Genèse, $7: 20$.

73. Genèse, $7: 24$.

74. Pierre Toussaint Marcel de Serres de Mesplès (1780-1862), géologue et naturaliste montpelliérain, fit paraître De la cosmogonie de Moïse comparée aux faits géologiques. Seconde édition, revue et considérablement augmentée en 1841.

75. Adolphe Brongniart, botaniste et paléontologue (1801-1876), fils du minéralogiste Alexandre Brongniart (1770-1847), neveu du chimiste Antoine-Louis Brongniart (1742-1804), est considéré comme l'un des précurseurs de la paléobotanique. Jean-Baptiste Léonce Élie de Beaumont (1798-1874), figure scientifique française de tout premier ordre, était géologue. Figuier, et Cotty après lui, s'inspirent de sa théorie de la formation des cordillères pour expliquer la création des montagnes. Edmond Hébert (1812-1890) était l'un des plus éminents géologues français de la seconde moitié du siècle, spécialiste du Jurassique, du Crétacé et de l'Ère tertiaire en France et en Angleterre. Le marquis Paul de Vibraye (Paul Hurault, 1809-1878), membre de la Société archéologique du Vendômois, de l'Académie des Sciences et de la Société géologique de France, possédait une riche collection paléontologique et préhistorique dans son domaine de Cheverny. Il 
pratiqua lui-même des fouilles géologiques, contribua à découvrir des outils datant de la Préhistoire et défendit Boucher de Perthes et son homme préhistorique. Édouard de Verneuil (1805-1873) était un géologue, disciple d'Élie de Beaumont, qui multiplia les expéditions autour du monde. Édouard Lartet (1801-1871) était un préhistorien et paléontologue. Il est considéré, avec Boucher de Perthes, comme l'un des fondateurs de la science préhistorique française. Albert Gaudry (1827-1908) était un spécialiste des fossiles de mammifères. Il défendait une forme de transformisme lamarckien chrétien, très différent de l'évolutionnisme de Darwin : selon lui, Dieu améliore les espèces au fil des siècles. Sa présence dans le poème de Cotty est très significative. Édouard Collomb (1801-1875) était un ingénieur chimiste de formation. À partir de 1840, il se consacra en amateur à la géologie, et en particulier aux anciens glaciers des Vosges, aux glaciers d'Europe et à la géologie de l'Espagne. Charles-Joseph Buteux (1794-1876), botaniste et géologue, membre de la Société géologique de France et de la Société Botanique de France, collabora avec Boucher de Perthes. Louis Frédéric Napoléon Bourgeois de Mercey (1838-1899?) était un géologue spécialiste de la Période quaternaire.

76. Charles Lyell (1797-1875), géologue britannique de premier ordre, fut un ardent défenseur de l'uniformitarisme. Ses Principes de géologie (1830-1833) demeurent une œuvre fondatrice pour la science géologique moderne. Il critique le transformisme lamarckien et ne reconnait pas la sélection naturelle darwinienne quoique, proche de Darwin, il apporta son appui à L'Origine des espèces. Cette attitude controversée vis-à-vis de l'évolutionnisme justifie sa présence dans le poème de Cotty, dont, de manière éloquente, Darwin est absent. John Wickham Flower (1807-1873) était un géologue et archéologue britannique amateur. Joseph Prestwich (1812-1896) était un géologue britannique spécialiste du Tertiaire. Il est considéré comme l'un des pionniers des études préhistoriques. Hugh Falconer (1808-1865) était un botaniste, géologue et paléontologue écossais. George Busk (1807-1886) était un chirurgien, zoologiste et paléontologue britannique, spécialiste des bryozoaires et des fossiles de vertébrés. John Evans (1823-1908), archéologue et géologue anglais, est l'un des pionniers des études préhistoriques. Cette liste de savants britanniques est justifiée dans ce texte patriotique dans la mesure où ces savants ont confirmé la découverte de l'homme préhistorique faite par Boucher de Perthes.

77. Jacques Boucher (de Crèvecœur) de Perthes (1788-1868) a posé les bases de la science préhistorique en France et dans le monde en faisant admettre l'existence, grâce à la découverte des bifaces d'Abbeville, de ce qu'il appelle «l'homme antédiluvien ». La mâchoire découverte en 1863, à Moulin-Quignon, fut en revanche dénoncée comme un faux, et la postérité ne revint pas sur l'authenticité de ce fragment humain, contrairement à ce que dit cotty dans la suite de son poème. Boucher de Perthes avait par ailleurs comme contradicteur le plus redoutable Élie de Beaumont, convaincu que l'homme ne pouvait pas avoir cohabité avec le mammouth. Cotty se permet ici une licence poétique en amputant son patronyme du -s final, qu'il rétablira quelques vers plus loin.

78. Sic.

79. Référence à l'Évangile de Matthieu, 13 : 30. Il s'agit de la parabole du semeur, que Cotty glose de manière discrète dans les vers qui suivent.

80. Cotty propose une version en vers du fragment $d u$ « Notre Père », prière que Jésus propose en modèle à ses disciples. Évangile de Matthieu, 6 :9/10. 


\section{RÉSUMÉS}

Cette édition vise à rendre facilement disponible un poème peu connu mais mis sur le devant de la scène par les récentes études épistémocritiques et notamment celles qui analysent l'inscription du savoir scientifique dans la poésie. Le texte de Cotty présente une conception originale de la biologie en diachronie qui est le reflet des paradoxes et conflits de son époque en matière d'évolutionnisme. Le texte annoté est enrichi d'une présentation fouillée qui aborde l'histoire du poème, sa génétique, sa poétique et ses influences ainsi que son apport dans l'histoire croisée des sciences et de la poésie.

This edition aims to easily make available a little-known poem yet put on the stage by recent epistemocritical studies, in particular by research which analyses the trace of scientific knowledge on poetry. The text of cotty produces an original approach of biology in diachrony which is the reflection of paradoxes and conflicts of its period in the area of evolutionism. The annotated text is enriched by an extensive introduction which addresses the story of the poem, its genetics, its poetics and its influences as well as its contribution in the overlapping history of sciences and poetry.

\section{INDEX}

Mots-clés : édition, poésie scientifique, épistémocritique, évolution, révolution

Keywords : edition, scientific poetry, epistemocritical, evolution, revolution

\section{AUTEURS}

\section{YOHANN RINGUEDÉ}

Laboratoire LISAA-EA4120, Université Gustave Eiffel, Université de Bâle 